\title{
XVII. Neues Aragonitvorkommen in Korlát, Comitat Nógrád ${ }^{1}$ ).
}

\author{
Von \\ A. Liffa in Budapest. \\ (Hierzu Tafel IX.)
}

Es sei in dem Folgenden kurz das bisher unbekannte Aragonitvorkommen in Korlát im Comitat Nógrád beschrieben, um dann zur krystallographischen Beschreibung des Aragonits überzugehen.

Auf das Vorkommen dieses untersuchten Materials bin ich ganz zufälligerweise gestoßen. Als nämlich im Laufe des vergangenen Sommers die Thököly-Straße der Haupt- und Residenzstadt Budapest neu geptlastert wurde, hatte ich Gelegenheit, täglich die in großer Menge aufgehäuften Basaltwürfel zu besichtigen. So fiel es mir auf, daß in dem Gesteine kleinere und größere Hohlräume in Erbsen- oder Haselnußform vorkommen, von denen einige mit schneeweißer kugeliger Mineralsecretion, andere hingegen mit feinen, haar- oder nadelförmigen kleinen Kryställchen ausgefüllt sind. Nachdem ich erfahren habe, daß das Gestein aus Korlát stammt, besuchte ich die Direction der $»$ Korláter Basaltsteinbruch-Actiengesellschaft , um mich in bezug der Herkunft des Gesteines vollständig zu überzeugen. Nachdem Herr Director A. Schweiger und Herr Directionsmitglied H. Reinisch die Herkunft des Gesteines bestätigten, erfuhr ich von den genannten Herren, daB ähnliche nadelförmige Krystalle - wohl selten auch in größerer Zahl in dem Basalte von Korlát vorzufinden sind. Eine kleinere Sammlung, die stets zu Handen war, wurde mir sogleich gefälligst zum Zwecke krystallographischer Untersuchung zugestellt, wofür es mir gestattet sei, auch an dieser Stelle den genannten Herren meinen höflichsten Dank darzubringen.

1) Vorgetragen in der Fachsitzung der ungarischen geologischen Gesellschaft am 2. Juni 1909. 
Auf diese Weise kam ich - außer dem eigengesammelten Material in den Besitz der aus vollständig ausgebildeten Krystallen bestehenden Stufen, die sich bei eingehender Untersuchung als Aragonit erwiesen haben.

Diese Stufen unterscheiden sich natürlich wesentlich von jenen, in den bearbeiteten Basaltwürfeln sichtbaren, erbsen- und haselnußförmigen Hohlräumen und deren Inhalt. Weil sie einesteils Bruchstücke großer Drusen bilden, anderenteils aber ihre Krystalle um vieles größer sind als jene, die in den erbsen- und haselnußähnlichen Hohlräumen sichtbar sind. Während diese kleinen Blasen meistenteils nur mit haarförmigen Kryställchen ausgefüllt sind, besitzen die Stufen wohlausgebildete, stattliche Krystalle, die eben deshalb für krystallographische Untersuchungen besonders geeignet waren. In den größeren Drusen befinden sich $5-7 \mathrm{~cm}$ lange, mit einander radial verwachsene Aggregate, die jedoch außer der mangelhaften Beschaffenheit ihrer Flächen, rauh, an den Spitzen abgebrochen und infolgedessen für krystallographische Untersuchungen gänzlich unbrauchbar sind.

In bezug auf die Größenverhältnisse der Hohlräume, respective um ihre maximale Ausdehnung zu beurteilen, sind nur wenig nähere Daten vorhanden. In der geodynamischen Sammlung der königlich ungarischen geologischen Reichsanstalt befinden sich drei Drusenstücke (geschenkt von der obengenannten Actiengesellschaft), welche einen Einblick in die Dimensionsverhältnisse gewähren. Das eine dieser Drusenstücke bildet eine Aushöhlung von beiläufig $10 \mathrm{~cm}$ Durchmesser, welche innerlich mit haselund wallnußähnlichem, weißem, kugeligem Aragonit ausgefüllt ist. Das andere Exemplar stellt dem Beschauer einen Schnitt durch einen beiläufig $10 \mathrm{~cm}$ langen, $4-5 \mathrm{~cm}$ hohen Hohlraum dar, in welchem außer dem kugelig ausgebildeten Aragonit noch auch beiläufig $5 \mathrm{~cm}$ lange, mit einander radial zusammengewachsene Krystallaggregate sichtbar sind. Das dritte Handstück ist ein mit Krystallen erfülltes Bruchstück einer Druse.

Wenn man nun die Stufen mit diesen Drusenstücken vergleicht, so ist aus der Form und Beschaffenheit der Stufen zu schließen, daß sie Teile um vieles größerer Hohlräume gewesen sind.

Der Aragonit wurde jedoch nicht nur in diesen Drusen, sondern auch in den Spalten des Basaltes ausgeschieden. Dies ist auf jenem großen, von diesem Fundort stammenden Basaltschaustücke nachzuweisen, dessen Oberfläche außer einer Aragonitkruste, mit kleineren bis größeren Krystallen überzogen und im Besitze der mineralogischen Sammlung des königlichen Josef-Polytechnikums ist.

Die Krystalle des Aragonits sind entweder mit einem Ende unmittelbar auf dem Basalt festgewachsen, oder sie stehen auf dem weißen sphäroïdisch ausgeschiedenen Aragonit, in welch letzterem Falle der Ausbildung der Krystalle die kugelige Secretion vorausgegangen war. 
Was nun die Entstehung des Aragonits betrifft, so ist es unzweifelhaft, daß derselbe secundären Ursprunges ist und hier - wie in den basischen Ergußgesteinen im allgemeinen immer - als Zersetzungsproduct auftritt ${ }^{1}$ ).

Indem ich nun zur krystallographischen Beschreibung des Aragonits übergehe, kann ich schon hier bemerken, daß mir ein ziemlich reiches Material zur Verfügung stand, indem die Anzahl der zur Untersuchung geeigneten Krystalle ca. 150-200 betrug. Es war jedoch nicht meine Absicht, dieselben alle einer Messung zu unterziehen, ich hatte es vielmebr darauf abgesehen, jene Typen festzustellen, welche für die Krystalle dieses Fundortes charakteristisch sind.

Ich hatte im ganzen 16 Krystalle gemessen und zwar acht einfache Individuen und acht Zwillinge; sämtliche sind in der Richtung der Verticalaxe langgestreckt und mit Ausnahme eines einzigen sind dieselben nur an einem Ende ausgebildet. Sie sind alle wasserklar und durchsichtig; doch finden sich außer diesen auf einer der Stufen auch solche, deren Oberfläche mit kleinen Aragonitkügelchen, oder mit einer weißen Kruste überzogen sind.

Die Größe der untersuchten Krystalle ist den oben erwähnten Vorkommnissen entsprechend verschieden. Die kleinsten (s. Fig. 2, Taf. IX), kaum stärker als ein Haar, sind $4 \mathrm{~mm}$ lang. Die größeren Krystalle (s. Fig. 1 und 3-3a) sind 6-8 $\mathrm{mm}$ lang, während ihre Dicke zwischen 1-2 mm schwankt. Ihre Flächen sind sehr lebhaft glänzend und geben gute Reflexe, mit Ausnahme der Prismenzone, deren Flächen bei weitaus den meisten Krystallen gerieft sind. Die Flächen der Form $m\{110\}$ sind bei manchen Individuen gegen die Verticalaxe geneigt und zeigen die Tendenz eine spitze Pyramide zu bilden. Diese Erscheinung ist mit Ausnahme einiger einfacher Krystalle besonders bei den Zwillingen zu beobachten, deren Prismenflächen, wie dies auch aus der $\pm d$-Rubrik der Winkeltabelle auf S. $255-256$ ersichtlich ist, eben deshalb keine verläßlichen Resultate lieferten.

Die untersuchten Krystalle zerfallen nach ihrer Entwickelung in zwei Gruppen: einfache und Zwillingskrystalle. Die einfachen Krystalle sind unter den kleineren Individuen überwiegend, die $Z$ willinge hingegen sind besonders unter den größeren Individuen häufig.

Von den bisher bekannten 107 Formen des Aragonits, welche im Jahre 1898 von Dr. Karl Zimányi zusammengestellt wurden 2), fand ich an dem untersuchten Materiale von Korlát folgende ausgebildet:

1) Vergl. Rosenbusch, Mikroskopische Physiographie usw., Stuttgart 1905, 2, 2. Teil, 1196.

2) K. Zimányi, Ủber den rosaroten Aragonit von Dognácska im Comitate Krassó-Szörény. Diese Zeitschr. 1899, 31, 353-371; und:

K. Zimányi, Adatok a dognácskai rózsaszinü aragonit kristálytani ismeretéhez (Természetrajzi füzetek $1899,22,452-472$ ). 


$\begin{array}{cc}b\{010\} & v\{031\} \\ m\{110\} & * D\{0.42 .19\} \\ * J\{11.11 .1\} & i\{021\} \\ p\{111\} & c\{011\} \\ s\{121\} & x\{012\}\end{array}$

Hieraus ist ersichtlich, daß der Aragonit von Korlát an Formen ziemlich arm ist. Die mit * bezeichneten Formen haben sich für den Aragonit als neu erwiesen; mit diesen zusammen beträgt die Zahl der Formen nun 109.

Von diesen zwei neuen Formen fand ich das Brachydoma $D\{0.42 .19\}$ an einem Zwillingskrystalle (s. Fig. 5, Taf. IX) als etwas matte, jedoch breite Fläche zwischen $i\{021\}$ und $b\{010\}$ ausgebildet.

Der steilen Pyramide $J\{11.11 .1\}$ entsprechen jene gegen die Verticalaxe geneigten Prismen, welche selten an den einfachen Krystallen, meistens aber an den $Z$ willingen auftreten.

Der Habitus der einfachen Krystalle wird von dem stets vorhandenen Prisma $m\{110\}$ bestimmt, nach welchem die Krystalle oft verzerrte sechseckige Polygone bilden. Das Brachypinakoid $\{010\}$ erreicht selten die Größe des Prismas und ist in der Regel durch mehr oder minder feine Riefung gekennzeichnet, weshalb dasselbe ähnlich wie die Flächen des Prismas, eine ganze Reihe von Signalen reflectiert. Die einfachen Krystalle lassen je nach ihrer Ausbildung und ihren Terminalflächen sehr verschiedene Typen unterscheiden.

Der erste Typus wird von der Combination der einfachsten Formen gebildet und besteht bloß aus den Flächen des Prismas $m\{110\}$, des Brachypinakoids $b\{010\}$ und des Brachydomas $k\{011\}$.

Der zweite Typus (s. Fig. 1) wird außer durch die soeben erwähnten Formen noch durch die Anwesenheit der kleinen, aber glänzenden Flächen der Pyramide $p\{111\}$ gekennzeichnet, denen sich an einem oder dem anderen Exemplare auch noch die Brachypyramide $s\{121\}$ und das Brachydoma $x\{012\}$ anschließt. Charakteristisch für die Krystalle dieses Typus ist es, daß die Grundpyramide $p\{111\}$ in der Richtung der krystallographischen Axe $a$, die Brachypyramide $s\{121\}$ und das Brachydoma $x\{021\}$ hingegen in der Richtung der Axe $b$ hemimorphe Ausbildung zeigen. Wie aus Fig. 1 ersichtlich, ist nämlich die Pyramide $s\{121\}$ in Form zweier schmaler, jedoch gut reflectierender Streifen blo $\beta$ in den zwei Oktanten des $+b$-Axenteiles entwickelt, während das Brachydoma $x\{012\}$ durch einen breiteren, gut reflectierenden Streifen bloß im positiven Oktanten des $b$-Axenteiles vertreten ist.

Zum dritten Typus (s. Fig. 2) gehören die langgestreckten haarfeinen Krystalle, an welchen das außerordentlich stark entwickelte Prisma 
$m\{110\}$ und Brachypinakoid $b\{010\}$ von den Flächen der beinahe vollkommen regelmäßig ausgebildeten Grundpyramide $p\{111\}$ und des Brachydomas $i\{021\}$ begrenzt wird. Bezeichnend für die Krystalle dieses Typus ist, daß nicht nur die Flächen der Pyramide und des Brachydomas, sondern - von den bisherigen abweichend - auch die Flächen der Prismenzone, trotz ihrer Schmalheit ausgezeichneten Glanz aufweisen, und demzufolge sehr gute Reflexe liefern.

Der vierte Typus (s. Fig. 3 und 3 a, Taf. IX) ist durch die flächenreiche Entwickelung der Brachydomenzone charakterisiert, indem an den hierher gehörigen Krystallen außer den Domen $x\{012\}$ und $k\{011\}$ auch noch die Brachydomen $i\{021\}$ und $v\{031\}$ anwesend sind. Von diesen Domen treten $k\{011\}$ und $i\{021\}$ an den untersuchten Exemplaren in beiden Oktanten auf, während $v\{031\}$ und $x\{012\}$ nur mit je einer Fläche vertreten. sind.

An einem der hierher gehörigen Krystalle hatte ich Gelegenheit, die geneigten Prismenflächen betreffend da sbeste Messungsresultat zu erhalten; aus welchem sich auf Grund des Tautozonalitätsgesetzes die Indices der Form

berechnen ließen.

Diese Form ist - wie schon oben erwähnt wurde - für den Aragonit neu; es zeigen jedoch die aus den Indices berechneten Werte den beobachteten Winkeln gegenüber eine das gewöhnlich zulässige Maß etwas übersteigende Abweichung. Den Grund hierfür müssen wir in der unvollkommenen Ausbildung der Flächen suchen, denn trotzdem dieselben besonders auf den $Z$ willingskrystallen mehrfach auftreten, geben sie nirgends tadellose Reflexe. Daß jedoch diese Form an den untersuchten Krystallen unzweifelhaft vorhanden ist, beweist am besten die Fig. 3a, in welcher sichtbar ist, daB das als schmaler Streifen auftretende Brachypinakoid die in der Fortsetzung der Prismenkante gelegene Polkante dieser spitzen Pyramide nicht mehr gerade, sondern schon keilförmig abstumpft.

Für diese Form habe ich folgende Winkelwerte erhalten:

$$
\begin{array}{rlr}
(11.11 .1):(110)= & \begin{array}{r}
\text { Gemessen: } \\
3^{0} 59^{\prime} 22^{\prime \prime}
\end{array} & \begin{array}{r}
\text { Berechnet: } \\
3^{0} 48^{\prime} 57^{\prime \prime}
\end{array} \\
:(111) & 321630 & 322655
\end{array}
$$

Wenn wir nun die einfachen Parameterverhältnisse der Pyramide \{11.11.1\} und den Umstand in Betracht ziehen, daß das ihr entsprechende Brachydoma $F\{0.11 .1\}$ schon seit längerer bekannt ist $\left.{ }^{1}\right)$, künnen

1) F. Stöber, Aragonit von Markirch und Framont (Mitteil. der geolog. Landesanstalt von Elaß-Lothringen 1898, 4, 141 und Ref. diese Zeitschr. 27, 532).

G. Cesàro erwähnt auch diese Form an den bei Long-Pré (Couthuin) gesammelten spießförmigen Zwillingen: Description des minéraux phosphatés, sulfatés et 
wir diese Form trotz der beträchtlicheren Abweichung zwischen den gemessenen und den berechneten Winkelwerten, dennoch - wenn auch mit Vorbehalt - als sicher bestimmte ansprechen.

Dieser Form steht bezüglich ihrer Parameterverhältnisse sehr nahe die von Schrauf am Aragonit von Dognácska beobachtete Pyramide $\left.\Theta\{10.10 .1\}^{1}\right)$; doch zeigen die für letztere berechneten Winkel eine noch größere Abweichung gegenüber den gemessenen Werten der Form $J\{11.11 .1\}$, und zwar:

$$
\begin{aligned}
& \text { Gemessen: } & & \text { Berecbnet: } \\
(11.11 .1):(110) & =3^{0} 59^{\prime} 22^{\prime \prime} & (10.10 .1):(110) & =4022^{\prime} \\
:(111) & =321630 & :(111) & =32 \quad 5
\end{aligned}
$$

Die Pyramide $J\{11.11 .1\}$ wurde wegen ihren allzu steilen Abschnitten in der perspectivischen Zeichnung nicht veranschaulicht, sondern nur im

\begin{tabular}{|c|c|c|c|c|c|c|c|c|c|c|c|}
\hline 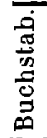 & 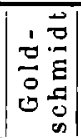 & Miller & $\varphi$ & $\varrho$ & $\xi_{0}$ & $r_{0}$ & $\xi$ & $\eta$ & $\begin{array}{c}x \\
(x: y)\end{array}$ & $y$ & $\begin{array}{l}d= \\
\operatorname{tg} \varrho\end{array}$ \\
\hline$J$ & 11.11 & $\{11.11 .1\}$ & $58^{0} 6^{\prime} 7$ & $86011^{\prime} 10^{\prime}$ & $85^{0} 0^{\prime} 3$ & $82048^{\prime} 35^{\prime \prime}$ & $\left|57053^{\prime} \mathbf{5} 5^{\prime \prime}\right|$ & $34049^{\prime} 8^{\prime \prime}$ & 12,7355 & 7,9266 & 15,000 \\
\hline
\end{tabular}
horizontalen Bilde auf Fig. 3a, Taf. IX, wo die Reductionsebene das Makropinakoid $a\{100\}$ ist.

Wenn wir nun die für das zweikreisige Goniometer bestimmten Projectionswinkel und die übrigen Hilfswerte dieser Form berechnen, erhalten wir folgende Resultate:

Es ist zu bemerken, daß ich mich bei der Berechnung dieser Werte auf die Grundwerte Goldschmidts ${ }^{2}$ ) stützte, um den vollen Einklang derselben mit den übrigen in den Winkeltabellen enthaltenen Angaben über Aragonit zu sichern.

Die Formen, welche sich am Aufbau der einfachen Krystalle beteiligen, sind in der Reihenfolge ihrer Größe die folgenden:

$$
m, J, k, b, i, v, p, s, x \text {. }
$$

Von diesen sind die Flächen von $k\{011\}$ als die glänzendsten und am besten reflectierenden hervorzuheben, welche sich an sämtlichen Krystallen durch ihre große Ausdehnung und durch ihre außerordentlich scharf be-

carbonatés du sol Belge (Mémoires de l'Académie royale de sciences, des lettres etc. Bruxelles, Sept., $53,1-134)$ und

G. Cesàro, diese Zeitschr. 31, 95.

1) A. Schrauf, Mineralogische Beobachtungen. I. Zwillingskrystalle des Aragonits (Sitzungsberichte der math.-naturwiss. Klasse der kais. Akad. der Wissensch. Wien 1870, 62, II. Teil, 734-845).

2) V. Goldschmidt, Krystallographische Winkeltabellen, Berlin 1897, 51-53. 
grenzten Reflexe auszeichnen. Ihre berechneten und gemessenen Winkelwerte weichen deshalb nur in Secunden von einander ab.

$$
\begin{array}{rlrl}
\text { Gemessen: } & \text { Berechnet: } \\
:(0 \overline{1} 1)= & 71033^{\prime} 43^{\prime \prime} & 71034^{\prime} 12^{\prime \prime} \\
:(012) & 15580 & 155755
\end{array}
$$

Ähnlichen Glanz und sehr scharfe Reflexe zeigen auch die verhältnismäßig kleinen Flächen von $p\{111\}$, welche ebenso wie die früher erwähnten auch mit den Werten Kokscharowsi) verglichen nur in den Secunden abweichen.

Die Formen $i\{021\}$ und $v\{031\}$ zeigen trotz ihrer wohl entwickelten Flächen schon schwächeren Glanz und schwächere Reflexe, während $s\{121\}$

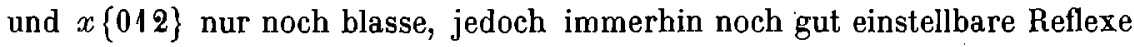
liefern, was durch die Kleinheit der Flächen und durch die geringe Intensität des reflectierten Lichtes leicht erklärlich ist.

Die gemessenen und berechneten Winkel der bisher schon besprochenen einfachen Krystalle, als auch der später zu behandelnden Zwillingskrystalle sind in folgender Tabelle mit einander verglichen zusammengestellt.

Als Basis der Berechnung dienten die Winkelwerte:

$$
\begin{aligned}
& (111):(011)=43011^{\prime} 0^{\prime \prime} \quad \text { und } \\
& (110):(010) \quad 58 \quad 4 \quad 30
\end{aligned}
$$

Es ist hier zu bemerken, daß der letztere Winkelwert nicht an der schlecht ausgebildeten Prismenzone der einfachen Krystalle, sondern an den ausgezeichnet spiegelnden und außerordentlich scharf begrenzte Signale liefernden Flächen eines $Z$ willings und mehrerer haardünner Krystalle bestimmt wurde.

$$
\begin{aligned}
& \text { Gemessene Winkel: Gemessen: (k. 2) } \left.n_{1}^{3} \text { ) } n_{2}{ }^{4}\right) \quad \pm d \text { Berechnet: } \\
& b: m=(010):(110)=58^{0} \quad 2^{\prime} 24^{\prime \prime} \quad 12 \quad 5 \quad 30 \quad 0^{0} 3^{\prime} \quad 5^{50} 4^{\prime} 30^{\prime \prime}
\end{aligned}
$$

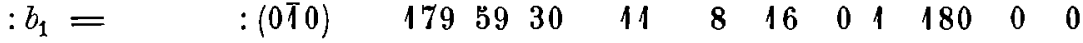

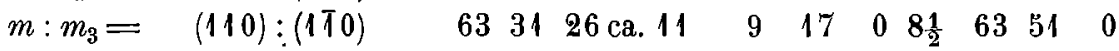

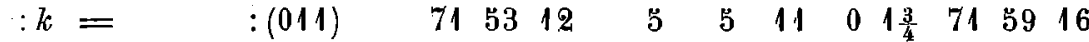

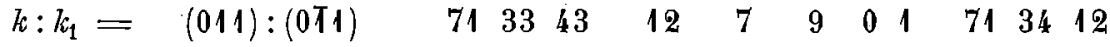

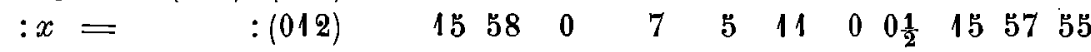

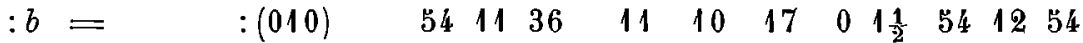

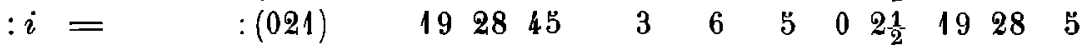

1) N. v. Kokscharow, Materialien zur Mineralogie Rußlands 6, 261.

E. S. D a n a, System of Mineralogy 1892, 282.

2) $\mathrm{Kr}$. = Zahl der Krystalle, an welchen die Winkel gemessen wurden.

3) $n_{1}=$ Zahl der bei der Bildung der arithmetischen Mittelwerte in Betracht gezogenen Kantenwinkel.

4) $n_{2}=$ Zahl der bel der Bildung der arithmetischen Nittelwerte nicht in Betracht genommenen Kantenwinkel. 
A. Liffa.

$$
\begin{aligned}
& \text { Gemessene Winkel: Gemessen: Kr. } n_{1} \quad n_{2} \pm d \text { Berechnet: } \\
& x: x_{1}=(012):\left(0 \bar{T}^{2}\right)=39^{0} 36^{\prime} 45^{\prime \prime} \quad 2 \quad 2 \quad 2 \quad 0^{0} 01^{\prime} 39^{0} 38^{\prime} 22^{\prime \prime}
\end{aligned}
$$

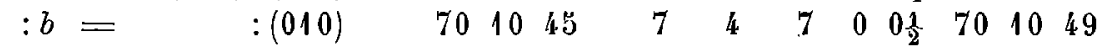

$$
\begin{aligned}
& i: i_{1}=\quad(021):(0 \overline{2} 1) \quad 1103010 \quad 4 \quad 3 \quad 2 \quad 0 \quad 1 \frac{1}{4} 1103022
\end{aligned}
$$

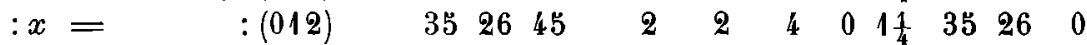

$$
\begin{aligned}
& : b=\quad:(010) \quad 34 \quad 4537 \quad 4 \quad 4 \quad 7 \quad 00 \frac{1}{2} \quad 34 \quad 44 \quad 49 \\
& : v=\quad:(031) \quad 10.4 \quad 0 \mathrm{ca} .1 \quad 1-2 \quad-95546 \\
& v: b=(031):(010) \quad 234530 \mathrm{ca} 1 \quad 1-24493 \\
& : k=\quad:(011) \quad 2931 \quad 0 \quad 1 \quad 1 \quad-\quad-\quad 292351 \\
& : k_{1}=\quad:(0 T 1) \quad 1012030 \mathrm{ca} .11-100583 \\
& p: k=(111):(011) \quad 431042 \quad 5 \quad 5 \quad 5 \quad 00 \frac{1}{2} \quad 43 \quad 11 \quad 0
\end{aligned}
$$

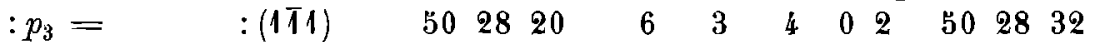

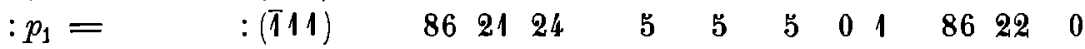

$$
\begin{aligned}
& : p_{2}=\quad:(T \bar{T}) \quad 1072630 \quad 1 \quad 1 \quad-\quad-\quad 1072816 \\
& : b=\quad:(010) \quad 64 \quad 4352 \quad 6 \quad 4 \quad 6 \quad 6 \quad 0 \quad 2 \frac{1}{2} \quad 65 \quad 45 \quad 44 \\
& : s=\quad:(121) \quad 18 \cdot 1130 \mathrm{ca} .3 \quad 3 \quad 2 \quad 1 \quad 0 \quad 9 \frac{1}{2} \quad 1843 \quad 24 \\
& s: b=(121):(010) \quad 4611 \quad 0 \quad 3 \quad 1 \quad 2 \quad-\quad 46 \quad 220 \\
& D: b=(0.42 .19):(010) \quad 32 \quad 6 \quad 0 \quad 1 \quad 1 \quad-\quad-\quad 32643 \\
& : i=\quad:(021) \\
& : k=\quad:(011) \\
& : x=\quad:(012) \\
& : c=\quad:(001) \\
& : D_{1}= \\
& :(0 . \overline{4} \overline{\mathbf{2}} .19) \\
& 24015 \quad 1 \quad 1-2386 \\
& \text { 22) } 724 \quad 1 \quad 1-20 \quad 22611 \\
& \begin{array}{llllllllll}
38 & 5 & 15 & 1 & 1 & - & - & 48 & 4 & 6
\end{array}
\end{aligned}
$$

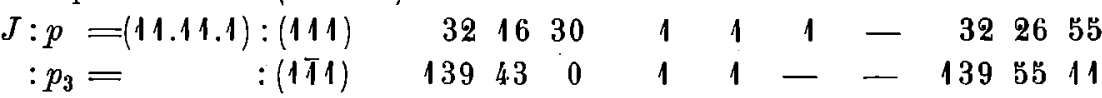

$$
\begin{aligned}
& : m_{3}=\quad:(1 T 0) \quad 175430 \quad 1 \quad 1-1761130 \\
& : J_{1}=\quad:(\bar{T} 1.11 .1) 116 \quad 6 \quad 0 \quad 1 \quad 1 \quad-\quad-1154436 \\
& : J_{3}=\quad:(11 . \overline{1} 1.1) \quad 631400 \quad 1 \quad 1 \quad-\quad-63.4130 \\
& : m=\quad:(110) \quad 35922 \quad 1 \quad 1 \quad-\quad-\quad 34857
\end{aligned}
$$

Aus den in Rubrik $\pm d$ angeführten arithmetischen Mitteln der Mittelwertdifferenzen der gemessenen Winkel ist ersichtlich, daß das Schwanken der Grenzwerte nur bei den Winkeln $m: m$ und $p: s$ beträchtlich ist, bei den übrigen hingegen als ziemlich geringfügig bezeichnet werden kann.

Hier kann ich noch erwähnen, daß ich mit Rücksicht darauf, daß mir für die Berechnung die oben angeführten vorzüglichen Werte zur Verfügung standen, es als nicht überflüssig erachtete auch das Axenverhältnis des Aragonits von Korlát zu bestimmen, hauptsächlich um feststellen zu können, inwiefern dasselbe mit dem Axenverhältnisse der von anderen ungarischen Fundorten beschriebenen Aragonite übereinstimmt, oder von demselben abweicht. Die Berechnung ergab für den Aragonit von Korlát folgendes Resultat:

$$
a: b: c=0,623050: 1: 0,720823 \text {. }
$$


Wenn wir diese Werte mit dem Axenverhältnisse des Aragonits von Dognícska, welches Melczer $\left.{ }^{1}\right)$ aus den von Zimányi ${ }^{2}$ ) gemessenen $k: k$, $k: b, m: m$ und $m: b$ Winkeln berechnete, und mit dem Axenverhältnisse des Aragonits von Úrvölgy (Herrengrund) vergleichen:

\begin{tabular}{|c|c|c|c|c|}
\hline Fundort: & $a: b: c$ & Autor: & \multicolumn{2}{|c|}{ Differenz: } \\
\hline & .0 & & $\breve{a}$ & $\dot{c}$ \\
\hline Dognácska & $0,6225: 1: 0,7203$ & Zimányi & 0,0005 & 0,0005 \\
\hline Úrvölgy & $0,6217: 1: 0,7207$ & Melczer & 0,0013 & 0,0001, \\
\hline
\end{tabular}

so sehen wir, daß in beiden Fällen sowohl die Axe $\breve{a}$ als $c$ kürzer sind als beim Aragonit von Korlát.

Mit den Axenverhältnissen anderer ausländischer Aragonite verglichen:

Fundort:

Leogang

Bilin

Framont

Mte. Ramazzo

Bilin

Hüttenberg-

Eisenerz
Autor:

$$
a: b: c
$$

$$
0,62234: 1: 0,72122
$$$$
0,62244: 1: 0,72057
$$$$
0,62247: 1: 0,71981
$$$$
0,62270: 1: 0,72070
$$$$
0,62280: 1: 0,72036
$$$$
0,62286: 1: 0,72077
$$

Differenz:

$\breve{a} \quad \dot{c}$

Buchrucker $\left.{ }^{3}\right) \quad 0,00071 \quad 0,00039$

Kokscharow $\left.{ }^{4}\right) \quad 0,00060 \quad 0,00025$

Stüber $\quad 0,00058 \quad 0,00101$

Negri6) $\quad 0,00035 \quad 0,00012$

Beckenkamp $\left.{ }^{7}\right) \quad 0,00025 \quad 0,00046$

Zepharovich $\left.{ }^{8}\right) \quad 0,00019 \quad 0,00005$

zeigt unser Aragonit mit jenem von Hüttenberg und Eisenerz die beste Übereinstimmung. Zepharovich acceptierte für diese Aragonite das von Miller ${ }^{9)}$ bestimmte Axenverhältnis.

Der Habitus der Zwillinge wird ganz ähnlich wie bei den einfachen Krystallen von dem vorherrschend entwickelten Prisma $m\{110\}$ bestimmt, nach dessen Flächen die Individuen zu Zwillingen, Drillingen und Viellingen

1) G. Melczer, Über den Aragonit von Úrvölgy (Herrengrund) (Diese Zeitschr. 1903, 38, 249-263) und Az úrvölgyi aragonitról (Math. és termtud. Értesitö 1903, 21, 236-254).

2) K. Zimányi, J. c.

3) L. Buchrucker, Die Mineralien der Erzlagerstätten vom Leogang in Salzburg. Diese Zeitschr. 1891, 19, 142.

4) N. v. Kokscharow, Materialien zur Mineralogie Rußlands 6, 264.

5) F. Stöber, Aragonit von Markirch und Framont (Mitteil. d. geolog. Landesanstalt von Elsaß-Lothringen 1898, 4, 119 und Ref. diese Zeitschr. 27, 532).

6) G. B. Negri, Über die Krystallformen des Aragonits vom Monte Ramazzo (Ligurien). Ref. diese Zeitschr. 1\$99, 30, 194 und Rivista di Min. e Crist. ital. di Panebianco 1896, 15, 57.

7) J. Beckenkamp, Zur Symmetrie der Krystalle. Diese Zeitschr. 1891, 19, 243.

8) V. v. Zepharovich, Mineralogische Mitteilungen. VI. Aragonitkrystalle von Eisenerz und Hüttenberg (Sitzungsber. d. kais. Akad. d. Wissensch. Wien 1875, 71, (I), 253).

9) Miller, Mineralogy 1852, ̈̈67. 
verwachsen sind. Diese $Z$ willinge lassen sich nach der Zahl der verwachsenen Individuen und nach ihrer Ausbildung in mehrere Typen einteilen, welche sämtlich für die Krystalle dieses Fundortes bezeichnend sind.

Zum ersten Typus gehören solche aus zwei Individuen zusammengesetzte Zwillinge, welche aus der Combination der einfachsten Formen: $m\{110\}, b\{010\}$ und $k\{011\}$ bestehen. Selten schlieBt sich diesen noch das Brachydoma $x\{012\}$ und die Pyramide $p\{111\}$ an; für diese beiden Formen ist es charakteristisch, da $\mathbf{B}$ erstere an den beiden in Zwillingsstellung befindlichen Individuen vollkommen symmetrisch ausgebildet ist, die letztere hingegen nicht.

Unter den hierher gehürigen Zwillingen hatte ich Gelegenheit, auch ein solches Exemplar zu untersuchen, welches aus zwei parallel verwachsenen Zwillingen zusammengesetzt war. Der eine dieser $Z$ willinge ist in Fig. 4, Taf. IX dargestellt; an dem zweiten, mit dem ersten parallel verwachsenen, gleich orientierten Zwilling fehlten die Flächen $p$.

In den $z$ weiten Typus sind jene $Z$ willinge eingereiht, bei denen die Flächen der Brachydomen $k\{011\}$ und $x\{012\}$ in dem einen Oktanten hervorragend ausgebildet sind und verzerrt erscheinen, im gegenüberliegenden hingegen nur als schmale Streifen auftreten (s. Fig. 6). Das perspectivische Bild dieser Zwillinge hat demzufolge ein eigentümliches, gegen eine Seite abfallendes Aussehen und es zeigt sich dieses schiefe Gefälle nach rechts oder links gerichtet, je nachdem die Flächen des Domas im positiven oder im negativen Oktanten überwiegend ausgebildet sind.

Den dritten Typus (s. Fig. 5) vertritt ein einziger Krystall von gedrungenem Habitus; im ganzen Material fand sich kein ähnlicher. Es ist dies ein Zwilling, welcher aus zwei Individuen besteht; ein besonderes Interesse verleiht $\mathrm{ihm}$ die Tatsache, daß er von einem geringen Defect abgesehen, an beiden Enden ausgebildet ist. In den horizontalen Projectionen, welche den oberen und unteren Pol des Krystalles veranschaulichen, gelangt die harmonische Ausbildung der Flächen an den beiden Enden klar zum Ausdruck, indem die zum $+b$-Axenteile gehörigen Flächen sowohl am oberen, als auch am unteren Ende in gleichem Maße vorherrschend, die zum - $b$-Axenteile gehörigen jedoch ganz untergeordnet zur EntwickIung gelangten. Die Anwesenheit einzelner Formen am negativen Ende der Verticalaxe wurde auch von Zimányi $\left.{ }^{1}\right)$ am Aragonit von Dognácska beobachtet, doch waren dieselben nur durch ein bis zwei Flächen vertreten.

Der Krystall dieses Typus ist ferner noch durch die ziemlich reich entwickelte Brachydomenzone gekennzeichnet, in welcher die Formen $k\{011\}, x\{012\}$ und $i\{021\}$ mit breiteren oder schmäleren Flächen auftreten. In dieser Zone fand ich auch eine für den Aragonit neue Form,

1) K. Zi mánỵi, l. c. S. 456, Fig. 2, 8, 9, 11, 14 und 16. 
welche als breite, jedoch etwas matte Fläche zwischen den Flächen der Formen $i\{021\}$ und $b\{010\}$ zur Entwicklung gelangte. Auf Grund des Tautozonalitätsgesetzes ließen sich nachstehende Indices berechnen:

$$
\{0.42 .19\} \text {. }
$$

Mit Rücksicht auf den Umstand, daß der aus diesen Indices berechnete Controllfehler nur etwa $0^{0} 1^{\prime} 13^{\prime \prime}$ beträgt und daB die Form zwar nur durch eine, jedoch wohlentwickelte Fläche vertreten wird und schließlich, daß die Indices ziemlich einfach sind, erscheint die Annahme dieser Form ohne Zweifel berechtigt. Den stichhaltigsten Beweis hierfür liefert die zwischen den gemessenen und berechneten Winkeln bestehende Übereinstimmung, welche aus der nachfolgenden Zusammenstellung ersichtlich ist:

$$
\begin{aligned}
& \text { Gemessen: Berechnet: }
\end{aligned}
$$

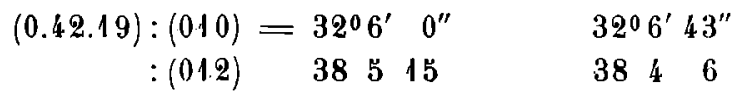

Wenn man aus den Grundwerten Goldschmidts ${ }^{1}$ ) die entsprechenden Positionswinkel und die übrigen Hilfswerte berechnet, ergeben sich

\begin{tabular}{|c|c|c|c|c|c|c|c|c|c|c|c|}
\hline 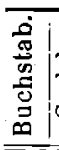 & 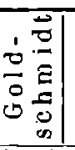 & Miller & $\varphi$ & $\varrho$ & $\xi_{0}$ & $\eta_{0}$ & $\xi$ & $\eta$ & $\begin{array}{c}x \\
(x: y)\end{array}$ & $y$ & $\begin{array}{l}d= \\
\operatorname{tg} \varrho\end{array}$ \\
\hline$D$ & $0 \frac{42}{15} \mid\{$ & $\{0.42 .19\}$ & $0^{0} 0^{\prime} 0^{\prime \prime}$ & $\left|57052^{\prime} 48^{\prime \prime}\right| 0$ & $000^{\prime} 0^{\prime \prime}$ & $57052^{\prime} 48^{\prime \prime}$ & $000^{\prime} 0^{\prime \prime}$ & $57^{052^{\prime}} 48^{\prime \prime}$ & 0,0000 & $1,5929 \mid$ & 1,5929 \\
\hline
\end{tabular}
folgende. Resultate:

Der vierte Typus (s. Fig. 7-8, Taf. IX) wird durch die Verwachsung von vier respective fünf Individuen gebildet. Die hierher gehörigen Viellinge sind von der Anzahl der verwachsenen Individuen abgesehen, in sehr ähnlicher Weise ausgebildet. Die vorherrschende Prismenzone wird bei den Vierlingen durch die Pyramide $p\{111\}$ und durch die Brachydomen $l\{\{011\}$ und $x\{012\}$, bei den Fünflingen außerdem noch durch die Brachypyramide $s\{121\}$ begrenzt. Es ist charakteristisch für die Krystalle dieses Typus, daß bei manchen Exemplaren die innerhalb liegenden Individuen in der Richtung der Zwillingsaxe stark zusammengedrückt sind, so daß sie nur als schmale Lamellen vorhanden sind. Sehr charakteristisch kommt dies am Vierling der Fig. 7 und am Fünfling der Fig. 9 zum Ausdrucke, in deren HorizontaJprojectionen jedoch diese Zwillingslamellen etwas breiter als in der Wirklichkeit dargestellt werden mußten, sonst hätten sie in den perspectivischen Bildern überhaupt nicht berücksichtigt werden künnen. Endlich sind die Krystalle dieses Typus noch dadurch charakterisiert, daß ihre Domenflächen an der einen Seite überwiegend ausgebildet sind, wodurch

1) V. Goldschmidt, l. c. S. 51 . 
den Zwillingen - ähnlich wie den Krystallen des zweiten Typus - ein eigentümlicher monokliner Ilabitus verliehen wird.

Die am Aufbau der Zwillingskrystalle beteiliglen Formen sind in der Reihenfolge nach ihrer Flächenausdehnung geordnet die folgenden:

$$
J, m, b, k, x, p, i, D, s \text {. }
$$

Von diesen sind als glänzendste und am schärfsten begrenzte Reflexe gebende Flächen besonders diejenigen der Formen $k\{011\}$ und $p\{111\}$ hervorzuheben, deren auffälliger Glanz die Orientierung der Individuen in vielen

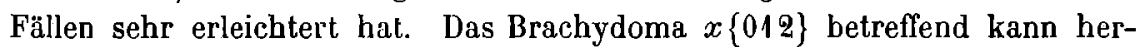
vorgehoben. werden, daß dasselbe, wie bei den einfachen Krystallen, so auch bei den Zwillingen in der Regel bloß durch eine, meist schmale Fläche vertreten ist (s. Fig. 6, 8 und 9, Taf. IX); wenn aber beide Flächen vorhanden sind, so ist eine derselben immer stärker ausgebildet als die andere (s. Fig. 4 und 5). Letzteres ist auch für das an den Zwillingen vorhandene Brachydoma $k\{011\}$ gültig. Das Brachydoma $i\{021\}$ fand sich mit hinreichend breiten Flächen bloß an dem in Fig. 5 dargestellten Zwilling vor, an dessen zwei Individuen diese Form zusammen mit fünf Flächen ausgebildet war. Die breiteren Flächen dieser Form waren sehr glänzend und gaben recht scharfe Reflexe; während die Reflexe der schmäleren Flächen

- schon weniger scharf erschienen. Die Brachypyramide $s\{121\}$ ist nur durch eine einzige Fläche am Fünfling der Fig. 9 vertreten und konnte trotz ihrer blassen Reflexe durch die in den Zonen [110:011] und [111:010] erzielten Messungsresultate mit völliger Sicherheit bestimmt werden. Die Prismenflächen zeigen eine vollkommene Ausbildung an den Krystallen der Figg. 4 bis 6 und 8; wohingegen an den größeren Zwillingen ihre Stelle von den sehr mangelhaft ausgebildeten Flächen der spitzen Pyramide $\{11.11 .1\}$ eingenommen wird.

Wenn wir nun zur Besprechung des Zwillingsgesetzes schreiten, können wir gleich feststellen, daß hier die Zwillingsebene $m\{110\}$ ist, welche zugleich auch die Verwachsungsebene ist. Was nun die Art und Weise der Verwachsung betrifft, muß vor allem entschieden werden, ob wir es hier mit Juxtaposition oder Penetration zu tun haben. Denn beim Aragonit treffen wir nach ein und demselben Gesetze - nicht selten sogar auf ein und demselben Krystalle - beide Verwachsungsweisen an ${ }^{1}$ ).

Die Entscheidung dieser Frage erheischt bei den aus zwei Individuen zusammengesetzten $Z$ willingen keine weitere Erörterung, denn die zwei

1) Vergl. diesbezüglich: F. Leydolt, Über die Structur und Zusammenselzung des prismatischen Kalkhaloides ... usw. (Sitzungsber. der math.-naturw. Klasse der kais. Akad. d. Wiss. Wien 1856, 19, 10-36).

G. Melczer, l. c.

A. Schrauf, l. c. 
Individuen sind, wie aus der horizontalen Projection der Fig. 4 ersichtlich, nach der Fläche $m\{110\}$ in Juxtaposition verwachsen. Bei den Vierlingen und Fünflingen müßten hingegen im Falle einer Penetration die Verwachsungsebenen unter einem gewissen Winkel gegeneinander geneigt und die Individuen cyklisch angeordnet sein, denn nur so wäre eine gegenseitige Durchdringung der Individuen möglich. Nun sind aber die Verwachsungsebenen der untersuchten Viellinge einander parallel, was sich gelegentlich der goniometrischen Messung dadurch verrät, daß die entsprechend gelegenen Flächen $m: \underline{m}$, von den Terminalformen aber die Flächen $k: \underline{k}$ an den polysynthetisch verwachsenen Individuen gleichzeitig reflectieren, wodurch es bewiesen ist, daß dieselben einen Winkel von $180^{\circ} \mathrm{mit}$ einander bilden.

An den in Zwillingsstellung befindlichen Individuen wurden nachstehende Winkel gemessen:

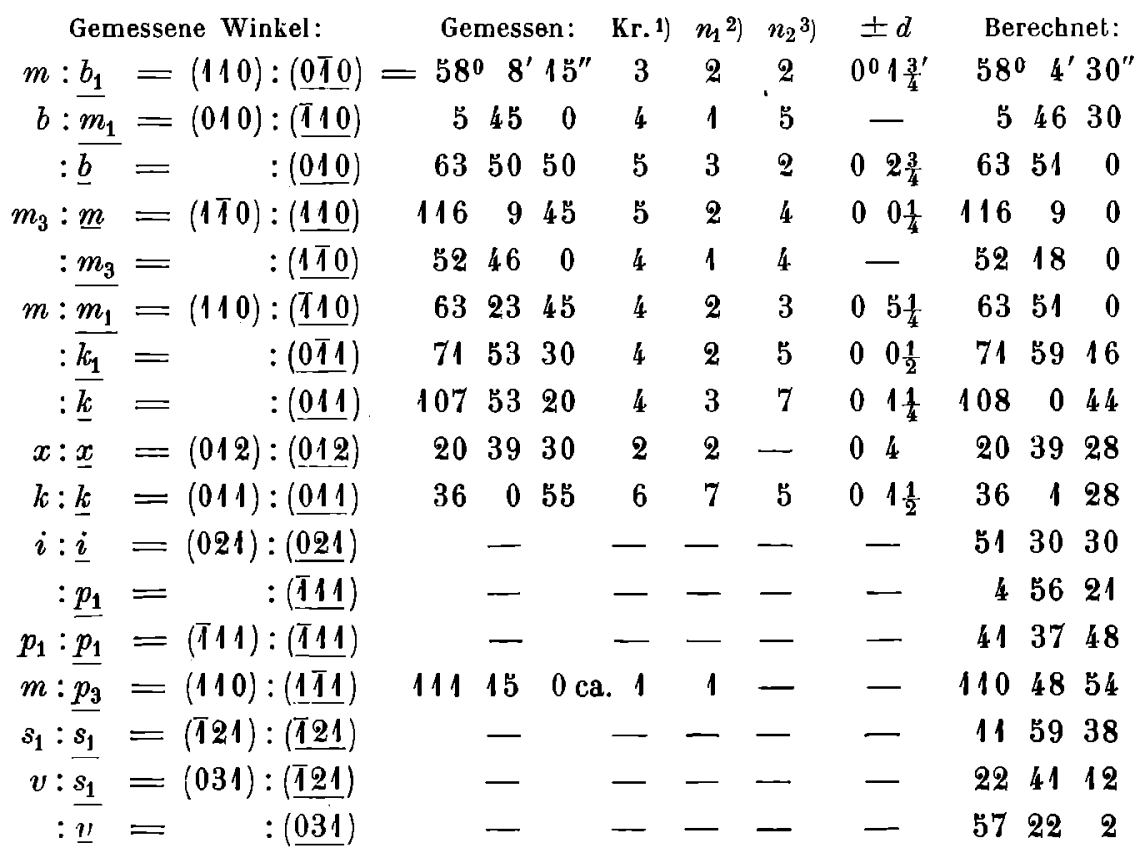

Wenn wir nun die unter den einfachen Krystallen und unter den Zwillingen festgestellten Typen vergleichend zusammenfassen, so können folgende gemeinsame Merkmale hervorgehoben werden: sämtliche Krystalle

1) $\mathrm{Kr}$. = Zahl der Krystalle, an welchen die Winkel gemessen wurden.

2) $n_{1}=$ Zabl der bei der Bildung der arithmetischen Mittelwerte in Betracht gezogenen Kantenwinkel.

3) $n_{2}=$ Zahl der bei der Bildung der aritbmetischen Mittelwerte nicht in Betrachl genommenen Kantenwinkel. 
sind in der Richtung der Verticalaxe gestreckt, besitzen größtenteils eine wohlentwickelte Brachydomenzone und auffallend glänzende Pyramidenflächen. Außerdem ist noch die $Z$ willinge respective Viellinge betreffend hervorzuheben, daß sämtliche nach $m\{110\}$ in Juxtaposition verwachsen sind, wobei häufig einzelne Individuen in der Richtung der Zwillingsaxe stark zusammengedrückt sind.

Zum Schlusse will ich noch bemerken, daß der größte Teil der Zeichnungen um ca. $18^{\circ}$ gedreht und ca. $8^{\circ}$ geneigt construiert wurde.

Ich kann die angenehme Pflicht nicht versäumen, dem Herrn Professor Dr. Franz Schafarzik an dieser Stelle meinen verbindlichsten Dank auszusprechen für seine Liebenswürdigkeit, mit welcher er es mir ermöglichte, meine Untersuchungen im Laboratorium seines Institutes durchzuführen, indem er mir die hierzu nötigen Apparate zur Verfügung stellte.

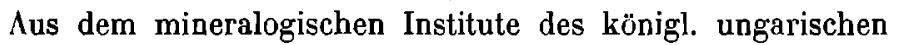
Josef-Polytechnikums in Budapest. 